\title{
The S68G polymorphism is a compensatory mutation associated with the drug resistance mutation K65R in CRF01_AE strains
}

Shengjia $\mathrm{Li}^{1,2,3+}$, Jinming Ouyang ${ }^{4 \dagger}$, Bin Zhao ${ }^{1,2,3}$, Minghui An ${ }^{1,2,3}$, Lin Wang ${ }^{1,2,3}$, Haibo Ding ${ }^{1,2,3}$, Min Zhang ${ }^{1,2,3}$ and Xiaoxu $\operatorname{Han}^{1,2,3^{*}}$

\begin{abstract}
Background: The rate of S68G mutation in human immunodeficiency virus type 1 (HIV-1) reverse transcriptase has increased and is closely related to the K65R mutation among CRF01_AE-infected patients who failed treatment. We aimed to explore the temporal association of S68G and K65R mutations and disclose the role of the former on susceptibility to nucleotide/nucleoside reverse transcriptase inhibitor (NRTI) and viral replication with the K65R double mutations among CRF01_AE-infected patients who failed treatment.

Methods: The occurrence of S68G and K65R mutations was evaluated among HIV-1 of various subtypes in the global HIV Drug Resistance Database. The temporal association of S68G and K65R mutations was analyzed through next-generation sequencing in four CRF01_AE-infected patients who failed treatment with tenofovir/lamivudine/ efavirenz. The impact of the S68G mutation on susceptibility to NRTI and replication fitness was analyzed using pseudovirus phenotypic resistance assays and growth competition assays, respectively.

Results: The frequency of the S68G mutation increased by $1.4-9.7 \%$ in almost all HIV subtypes and circulating recombinant forms in treatment-experienced patients, except subtype F. The S68G mutation often occurred in conjunction with the K65R mutation among RTI-treated patients, with frequencies ranging $21.1-61.7 \%$ in various subtypes. Next-generation sequencing revealed that the S68G mutation occurred following the K65R mutation in three of the four CRF01_AE-infected patients. In these three patients, there was no significant change detected in the half maximal inhibitory concentration for zidovudine, tenofovir, or lamivudine between the K65R and K65R/ S68G mutations, as demonstrated by the phenotypic resistance assays. Virus stocks of the K65R and K65R/S68G mutations were mixed with 4:6, 1:1, and 9:1 and cultured for 13 days, the K65R/S68G mutants outgrew those of the K65R mutants irrespective of the input ratio.
\end{abstract}

(Continued on next page)

\footnotetext{
* Correspondence: hanxiaoxu@cmu.edu.cn

${ }^{\dagger}$ Shengjia Li and Jinming Ouyang contributed equally to this work.

${ }^{1} \mathrm{NHC}$ Key Laboratory of AIDS Immunology (China Medical University),

Department of Laboratory Medicine, The First Affiliated Hospital of China

Medical University, Shenyang 110001, China

${ }^{2}$ National Clinical Research Center for Laboratory Medicine, Shenyang

110001, China

Full list of author information is available at the end of the article
}

C) The Author(s). 2020 Open Access This article is distributed under the terms of the Creative Commons Attribution 4.0 International License (http://creativecommons.org/licenses/by/4.0/), which permits unrestricted use, distribution, and reproduction in any medium, provided you give appropriate credit to the original author(s) and the source, provide a link to the Creative Commons license, and indicate if changes were made. The Creative Commons Public Domain Dedication waiver (http://creativecommons.org/publicdomain/zero/1.0/) applies to the data made available in this article, unless otherwise stated. 
(Continued from previous page)

Conclusions: S68G may be a natural polymorphism and compensatory mutation of K65R selected by NRTIs in the CRF01_AE strain of HIV-1. This mutation does not affect susceptibility to NRTI; however, it improves the replication fitness of K65R mutants. This study deciphers the role of the S68G mutation in the HIV reverse transcriptase of the CRF01_AE strain and provides new evidence for the interpretation of drug-resistant mutations in non-B subtypes of HIV-1.

Keywords: CRF01_AE, Polymorphism, Drug resistance, Replication fitness

\section{Background}

Interpretation of human immunodeficiency virus type 1 (HIV-1) drug resistance mutations (DRMs) is mainly based on phenotypic and genotypic studies of subtype-B, which accounts for $\sim 12.1 \%$ of HIV infection cases worldwide [1]. Numerous patients infected with non-B subtype HIV-1 in developing countries receiving antiretroviral therapy (ART) require more precise interpretation of DRMs [2, 3]. The high genetic diversity of HIV1 poses great challenges to the interpretation of the genotyping of drug resistance.

Several studies have suggested that DRMs may not be fully consistent among different subtypes of HIV-1. For instance, HIV-1 strains of different subtypes can develop various "signature" DRMs under the same ART regimen $[4,5]$. Some studies have suggested that natural polymorphisms of the non-B subtypes of HIV-1 change their susceptibility to ART independently or in combination with other mutations [6, 7]. Additional studies are warranted to elucidate the role of natural polymorphisms and certain treatment-associated mutations in the non-B subtypes of HIV-1.

CRF01_AE is the first circulating recombinant form of HIV-1 identified worldwide. It originated from Central Africa, and accounts for $4.6 \%$ of the total number of HIV-1 infections worldwide [8, 9]. CRF01_AE may have distinctive characteristics on pathways related to natural polymorphisms and the development of DRM [10-12]. A preliminary study on CRF01_AE-infected patients, conducted in China by our research team, found that the frequency of the S68G mutation showed a $5.5 \%$ increase in patients who failed treatment. Moreover, the S68G mutation demonstrated a close link with the K65R mutation (unpublished data). The role of the S68G mutation in drug resistance and its relationship with the K65R mutation has yet to be elucidated. Thus, we conducted this study to: 1) explore the frequency of S68G mutation and K65R/S68G double mutation occurrence among various subtypes of HIV-1 worldwide; 2) ascertain the temporal association of S68G and K65R mutations among patients infected with CRF01_AE who failed treatment; and 3) understand the role of the S68G mutation in susceptibility to NRTI and viral replication when combined with the K65R mutation.

\section{Methods \\ Occurrence of S68G and K65R/S68G mutations among various subtypes of HIV-1}

The prevalence of S68G mutation and K65R/S68G double mutation among various subtypes of HIV-1 was analyzed in reverse transcriptase inhibitor (RTI)-naive and RTI-treated individuals from the HIV Drug Resistance Database of Stanford University (https://hivdb.stanford.edu/cgi-bin/MutPrevBySubtypeRx.cgi; version of 19 April 2019). For patients having more than one isolates with the same mutation are counted once. Mutations occurring in $\geq 1 \%$ and at least two individuals were included in the analyses. All sequences with a mixture of mutations were excluded from the analyses.

\section{Study participants and sample collection}

Four patients infected with CRF01_AE who failed treatment and carried the K65R and S68G double mutations were selected from a large-scale long-term ART cohort at the First Affiliated Hospital, China Medical University (Shenyang, China) (Additional file 1: Figure S1). The patient identification numbers were 301635, 301770, 301844 , and 302335. The treatment regimens were tenofovir (TDF), lamivudine (3TC), and efavirenz. Genotypic testing for drug resistance and the viral load of plasma was performed at baseline, and at 6, 7, 10, and 12 months after treatment. Thereafter, all four patients changed treatment regimens. Among the four patients, 301635 and 302335 were followed up four times within 6-7 months post ART, while 301770 and 301844 were followed up five times within 10-12 months post ART. The study protocol was approved by the Ethics Committee of the First Affiliated Hospital of China Medical University. Written informed consent was provided by all patients.

\section{Extraction, amplification, and sequencing of HIV-1 DNA}

Viral RNA was extracted from plasma using a QIAamp RNA Blood Mini kit (Qiagen, Stanford, VA, USA). The partial pol gene (145 bp) was amplified using primers 503b-F (5' -CAAAAATTGGGCCTGAAAATCCATA-3') and 52r-R (5'-TGTGGTATTCCTAATTGAACTT CCCA-3') through nested polymerase chain reaction (PCR). PCR products were purified with an Agencourt AMPure $^{\mathrm{TM}}$ kit (Beckman Coulter, Fullerton, CA, USA) 
and quantified using a Qubit ${ }^{\circ}$ dsDNA BR Assay kit (Life Technologies, Carlsbad, CA, USA). Subsequently, the PCR products were evaluated with a Bioanalyzer (2100; Agilent Technologies, Santa Clara, CA, USA) to control the size of the amplified fragments, and a library was constructed based on an input of 130-150 ng. A TruSeq $^{\mathrm{m}}$ Nano DNA HT Sample Preparation kit was employed for the construction of a DNA library (Illumina, San Diego, CA, USA). The quality of library construction was monitored using a DNA 7500 kit (Agilent Technologies). The standardized libraries were incorporated into 50\% quality-controlled PHIX Libraries (Miseq phix control v3; Illumina). Sequencing was performed after the qualification of all operations.

The FASTQC (version 0.11.5) software was used to evaluate the sequencing quality of the paired-end FASTQ sequence files. MiSeq data analysis was performed using HyDRA Web, an open web portal that offers an automated pipeline for the analysis of nextgeneration sequencing-derived data related to HIV drug resistance. Advanced options for data quality assurance, filtering, variant calling, and reporting thresholds were modified to customize the analysis. Reads were filtered using a minimum quality score of Q30 and $50 \mathrm{bp}$ in length, an error rate of 0.0021 for the MiSeq platform, a minimum variant quality of Q30, a minimum read depth of $100 \times$, and a minimum allele count of five. HIV-DRMs detected with a frequency $>1 \%$ were reported based on the default HyDRA Web Mutation Database. This database is a combination of the Stanford 2015 list of HIV-1 DRMs with added annotations from the World Health Organization 2009 list of mutations for the surveillance of transmitted HIV drug resistance [13].

\section{Construction of a mutant clone and phenotypic resistance assay}

Protease (PR) genes (codons 1-99) and reverse transcriptase (RT) genes (codons 1-240) were amplified from viral RNA obtained from patients infected with CRF01_AE using nested PCR using primers Round2-F (5'-ATAGCCAAAAATTGCAGGGCCCCTAG

RAAAAAG-3') and Round2-R (5'-GTCCTTCCTT TCCACATTTCCA-3'). Patient-derived PCR products containing the $\mathrm{K} 65 \mathrm{R} / \mathrm{S} 68 \mathrm{G}$ double mutations were cloned into a pNL4-3- $\Delta \mathrm{E}$-Luc plasmid through a ApaI/ AgeI double-enzyme digestion and T4 ligation strategy to produce the pNL4-3- $\Delta$ E-Luc K65R/S68G clone. Subsequently, the S68G mutation was reversed to wild-type through in vitro site-directed mutagenesis using primers G68S-F (5'-AAGAGAAAGGACAGTACCAAATGGAGAAAG-3') and G68S-R(5'-TCTCCATTTGGTAC TGTCCTTTCTCTTTAT-3') to produce the pNL4-3$\triangle \mathrm{E}$-Luc K65R clone. Pseudoviruses were packaged in $293 \mathrm{~T}$ cells with plasmid VSVG and co-transfection of the K65R/S68G clone or K65R/S68G clone [14]. The titers of viral stocks were determined according to the Spearman-Karber method [15].

The susceptibility of the virus to AZT, 3TC, and TDF was calculated as the half maximal inhibitory concentration (IC50). All experiments were conducted in triplicate. The IC50 of the mutant virus was compared with that of the fully susceptible virus (wild-type pseudovirus NL4-3- $\Delta \mathrm{E}$-Luc). The degree of resistance of the mutant pseudoviruses was determined by calculating the fold change (FC) in IC50 compared with that of the wildtype pseudoviruses.

\section{Growth competition assay}

PR genes and RT genes of the pNL4-3-wildtype plasmid were replaced by patient-derived genes containing K65R/ S68G double mutations through an ApaI/AgeI doubleenzyme digestion and T4 ligation strategy to produce the pNL4-3-wildtype K65R/S68G mutant infectious clone. Subsequently, the S68G mutation was reverted to wild type through in vitro site-directed mutagenesis to produce the pNL4-3-wildtype K65R mutant infectious clone. The K65R mutant and K65R/S68G mutant viral stocks were produced via transfection of $293 \mathrm{~T}$ cells, and cultured at $37^{\circ} \mathrm{C}$ in an atmosphere of $5 \% \mathrm{CO}_{2}$ for $48 \mathrm{~h}$. The supernatant was collected for viral titration. The K65R/S68G mutants and K65R mutants viral stocks were diluted and mixed at a 4:6, 1:1, and 9:1 ratio, respectively. Peripheral-blood mononuclear cells $\left(3 \times 10^{5}\right)$ obtained from healthy donors were infected with the same virus titers (multiplicity of infection $=0.05$ ) from each of the competing viruses. All experiments were conducted in triplicate. Viral fitness was determined through full pairwise competitions between all combinations of viruses. On days $3,5,7,10$, and 13 , half of the culture supernatant was harvested, and viral RNA was extracted. The viral pol gene was amplified and sequenced for Sanger sequencing. The ChromatQuan Internet tool was used to calculate the viral ratio at each time point [16].

\section{Statistical analysis}

The chi-squared test was used to evaluate the frequency of S68G mutation between RTI-naive and -treated individuals, and the K65R/S68G double mutation among HIV-1 subtypes. The nonparametric t-test was used to analyze differences in FC between $\mathrm{K} 65 \mathrm{R}$ alone and K65R/S68G double mutants. A $p<0.05$ denoted statistical significance.

\section{Results}

Frequency of the S68G mutation increased significantly in RTI-treated patients and commonly co-occurred with the K65R mutation among CRF01_AE strains

A total of 172,639 RTI-naive and RTI-treated individuals infected with HIV-1 in the HIV Drug Resistance 
Database of Stanford University were analyzed in this study: subtype A $(n=12,415)$, subtype B $(n=83,006)$, subtype C $(n=36,436)$, subtype $\mathrm{D}(n=3705)$, subtype $\mathrm{F}(n=2418)$, subtype G $(n=4638)$, CRF01_AE $(n=20,507)$, and CRF02_ AG $(n=9514)$. Prior to ART, the lowest and highest prevalence of the S68G mutation were reported in subtype $\mathrm{G}$ $(1.3 \%, n=1564)$ and CRF01_AE $(7.3 \%, n=14,336)$, respectively. Among RTI-treated patients, the prevalence of the S68G mutation increased significantly in almost all subtypes $(p<0.05)$, except subtype $\mathrm{F}$ that exhibited a slight decrease. CRF01_AE viruses demonstrated a 9.7\% increase in S68G mutation between RTI-treated and RTI-naive individuals, the greatest increase observed among all subtypes (Additional file 2: Table S1). Surprisingly, the S68G mutation showed a high tendency to coincide with the K65R mutation in various subtypes. The prevalence of the K65R/ S68G double mutation among RTI-treated patients of various subtypes ranged from $21.1 \%(4 / 19)$ in subtype $\mathrm{F}$ to 61.7\% (142/230) in CRF01_AE (Additional file 3: Table S2).

S68G mutation is often selected on the basis of the K65R mutation

An average of 260,000 high quality reads (> Q30) were obtained from each of the four cases, which comprehensively reflected the sequence characteristics of the mutant. Among the three patients infected with CRF01_AE not carrying the natural polymorphism S68G prior to ART, The K65R mutation was detected in patient-302335 as the dominant mutant quasispecies 3 months post-ART, accounting for $92.99 \%$, which remained at a high level (94.63\%) when tested 6-months post-ART; at that time, the S68G mutation was detected among $51.14 \%$ quasispecies. The other two patients (301770 and 301844) had the predominant quasispecies carrying the K65R mutation at 3-6 months post ART (accounting for 95.38 and $92.51 \%$, respectively). The S68G mutation occurred at 77.52 and $59.19 \%$ respectively. In further follow-up time points (at 7-12 months post ART), almost all quasispecies carried the K65R/S68G double mutation in these two patients. Unlike the above three patients, patient 301635 carried the S68G mutation as the predominant natural polymorphism, appearing in $>90 \%$ of the quasispecies prior to ART. However, the K65R mutation was not detected until 7 months post ART, when only $11.28 \%$ of the quasispecies were found to have the K65R mutation (Fig. 1).

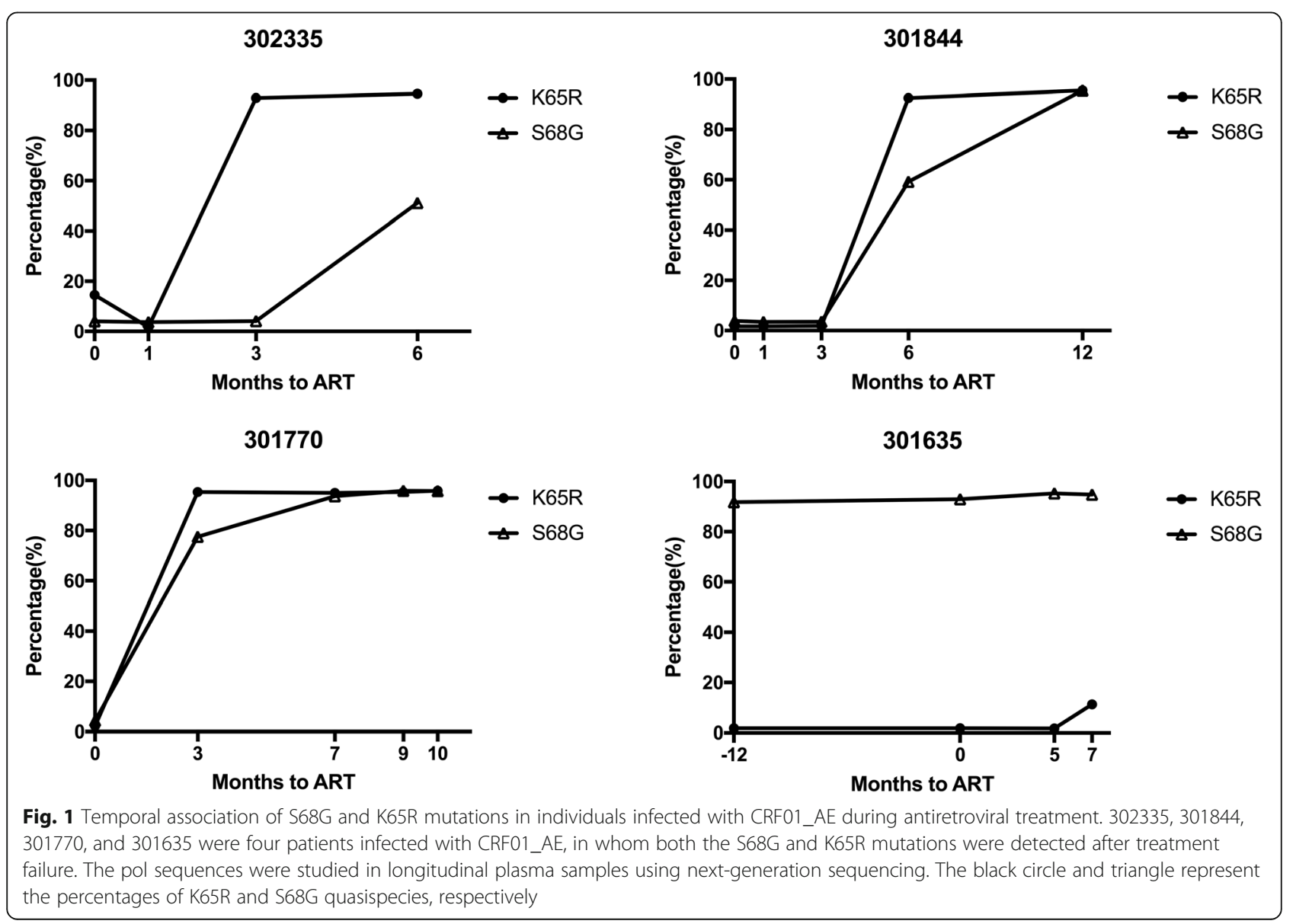


S68G mutation does not decrease the drug susceptibility of the K65R mutation to AZT, TDF, or 3TC

The frequency of the K65R mutation in patient 301635 was insufficient to select clones of the K65R/S68G double mutation; hence, this patient was not included in subsequent analyses. Therefore, clones of the K65R/ S68G double mutation and K65R mutation from the other three patients were used for analyses of phenotypic drug resistance. The viruses harboring the K65R/S68G double mutation in patients 301770, 301844, and 302335 exhibited a FC to AZT of $0.562 \pm 0.067,1.483 \pm 0.529$, and $1.359 \pm 0.137$, respectively. These values were not significantly different from those of the K65R mutation (FC: $0.925 \pm 0.214,1.262 \pm 0.618$, and $0.793 \pm 0.09$, respectively; $p>0.05$ ). Moreover, there were no significant changes observed in the degree of resistance to 3TC or TDF (Fig. 2).

\section{S68G mutation compensated for the replication fitness of} the K65R mutation

Virus stocks of the K65R and K65R/S68G mutations were mixed with different input ratios and cultured for 13 days. At the end of culture, the K65R/S68G double mutation strains outgrew those of the K65R mutation irrespective of the input ratio. In the 4:6 input group, the composition of K65R/S68G mutants increased from 60 to $88 \%$. In the $1: 1$ input group, the composition of K65R/S68G mutants increased from 50 to $84 \%$. In the 9: 1 input group, the composition of K65R/S68G mutants increased from 10 to $62 \%$ (Fig. 3).

\section{Discussion}

In this study, we found that the S68G mutation was a common natural polymorphism in various subtypes of HIV-1, predominantly among CRF01_AE strains. The S68G mutation often occurred following the K65R mutation in patients infected with CRF01_AE who failed treatment. However, the pre-existence of the S68G natural polymorphism may not accelerate the occurrence of the K65R mutation. Although S68G did not directly alter the drug susceptibility of the virus, it compensated for the loss of replication fitness associated with the K65R mutation in CRF01_AE HIV-1.

We found that the mutation rate of S68G increased in almost all HIV-1 subtypes in drug-experienced populations, with the greatest increase noted among CRF01_ AE strains. This finding is supported by several studies on non-subtype B HIV-1 $[17,18]$. Based on the available evidence, it is suggested that S68G may be new mutation associated with the development of drug resistance.

Moreover, previous studies showed that patients infected with subtype B and non-B subtype HIV-1, who failed ART and developed the K65R mutation, also acquired the S68G mutation [18-22]. However, the unexpected high frequency of the S68G mutation in combination with the K65R mutation in the CRF01_AE strain has not been previously reported. Several studies have examined the evolution of the K65R and S68G mutations using standard testing for HIV drug resistance or molecular clones. However, the conclusions drawn from these investigations have been controversial. Ross et al. reported that mutations at position 68 followed the initial selection for K65R and M184 V mutations [23]. Roge et al. found that five patients with the wild-type virus at baseline developed K65R at the time of failure, and four of those patients also acquired the S68G mutation [20]. However, Wirden et al. found that the S68G mutant was the dominant quasispecies, whereas K65R/S68G double mutant was the minor quasispecies. This finding suggested that the S68G mutation may not be linked to the K65R mutation [24]. In the aforementioned studies, variants with a frequency $<20 \%$ of the viral population could hardly be detected due to the limitation of Sanger sequencing, molecular clones, and PCR bias [25]. In this study, we performed next-generation sequencing to explore the evolutionary relationship between $\mathrm{K} 65 \mathrm{R}$ and S68G mutations using longitudinal samples. This approach can detect viral quasispecies with frequencies as

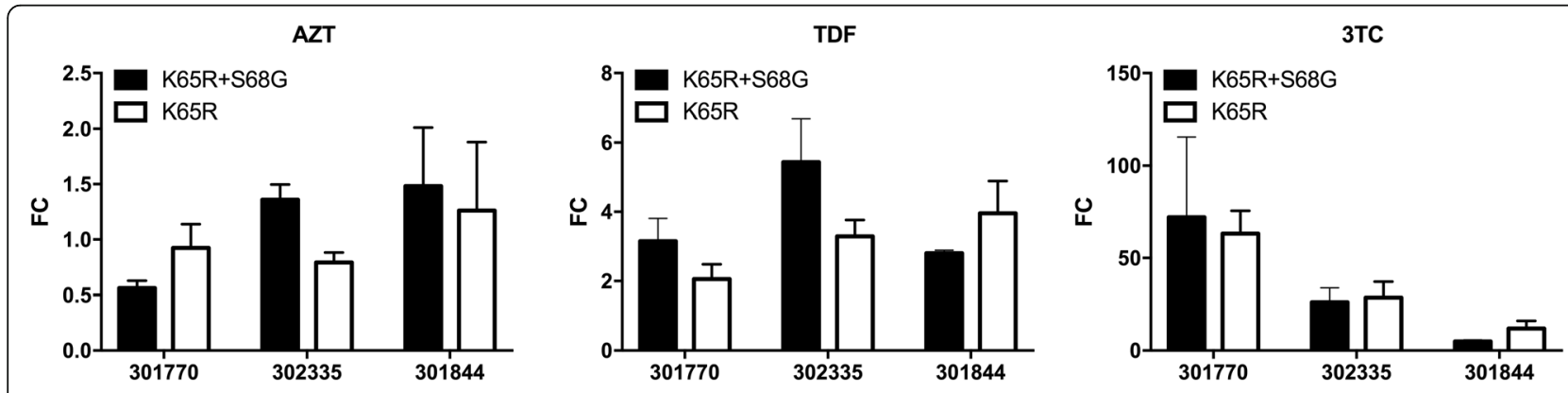

Fig. 2 Pseudovirus-based phenotypic resistance assay for AZT, TDF, and 3TC. Black columns and white columns represent the K65R/S68G double mutation and K65R mutation pseudoviruses with mutant pol gene sequences obtained from patients, respectively. FC, fold change in IC50 compared with the wild-type pseudoviruses; AZT, zidovudine; TDF, tenofovir; 3TC, lamivudine. Data were averaged from at least three independent experiments 

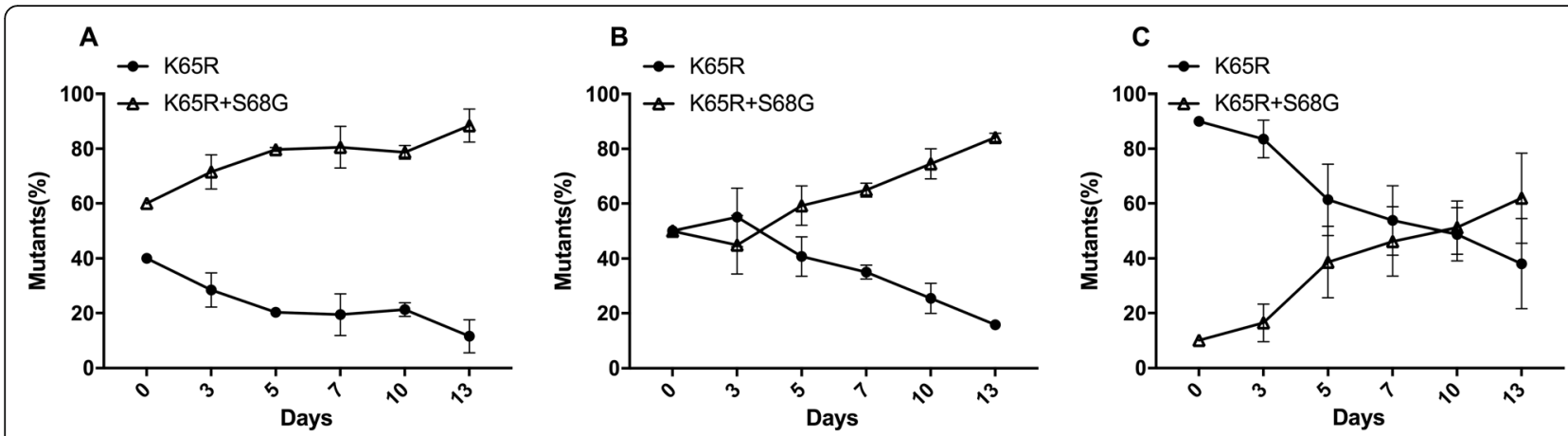

Fig. 3 Growth competition assay for the K65R and K65R/S68G infectious clones. The infectious clones of K65R and K65R/S68G obtained from patients were co-cultured for 13 days in PBMCs with the following input ratios for K65R vs. K65R/S68G: 4:6 (a), 1:1 (b), and 9:1 (c). Viral RNA from culture supernatants was used to amplify target regions of pol genes, and these regions were subsequently sequenced. ChromatQuan (http:// indra.mullins.microbiol.washington.edu/cgi-bin/chromatquant.cgi) was used to calculate the viral percentages at each time point. The average of three independent experiments is shown, with error bars representing the SD

low as $1 \%$, and generate more data than Sanger sequencing (1000s-10000s magnitude) [26]. Therefore, we disclosed the temporal association between these two mutations among patients infected with CRF01_AE who failed treatment. Our results demonstrated that the frequency of the K65R/S68G double mutation was lower than that of the K65R mutation in vivo. Moreover, a time lag was detected between the occurrence of the S68G and K65R mutations. Hence, the K65R mutation may be induced first as the major mutation under the pressure of ART, while S68G may be an accessory mutation of K65R that is subsequently caused on the basis of the K65R mutation. We sought to investigate whether the preexistence of the S68G natural polymorphism prior to treatment could assist the development of the K65R mutation. We noticed that, in one patient with > $90 \%$ quasispecies carrying the S68G mutation prior to treatment, the K65R mutation was not detected until 7 months post ART. This timing was later than that observed for the remaining three patients not carrying the S68G polymorphism prior to treatment, suggesting that the S68G mutation may not accelerate the development of the K65R mutation.

We subsequently examined the phenotype of S68G. Svarovskaia evaluated the role of the S68G in combination with $\mathrm{K} 65 \mathrm{R}$ in subtype B. The analysis revealed that S68G mutations partially restored the replication defect associated with the K65R mutation; however, it did not cause significant change in resistance of HIV-1 to NRTIs [27]. In this study, we focused on the phenotype of S68G in the CRF01_AE virus. CRF01_AE virus had an obvious genetic difference on the pol gene compared with that of the subtype B virus. In addition, it occurred markedly more often in both treatment-naive patients and those who failed treatment than that of the subtype B virus. We used patient-derived pol gene fragment replacement for the construction of the mutant plasmid instead of site-directed mutagenesis on the basis of the subtype B standard plasmid to maintain the genetic background of the pol gene of CRF01_AE. We disclosed the minor role of the K65R mutation with and without S68G in susceptibility to NRTI. However, the S68G mutation helps to compensate the loss of replicative fitness, which is similar to that of subtype B.

The $\mathrm{K} 65$ residue is located on the key position of the deoxynucleoside triphosphate binding pocket and associated with the development of resistance to nucleoside analogs and polymerase fidelity [28]. This residue confers resistance to numerous NRTIs by reducing the efficiency of NRTI incorporation. It has also been speculated that the K65R substitution improves the fidelity of the polymerase, thereby reducing the occurrence of several types of RT errors, including substitution and frameshift mutations [29-31]. It has been reported that the increase in polymerase fidelity may render the viral population less able to adapt to drug treatment and immune surveillance, and reduce replication fitness [32]. Given the importance of $\mathrm{K} 65$ residues in the RT function of HIV-1, and the observed reduced fitness of the K65R mutant, the virus must evolve compensatory mutations against the impairment. With regard to patients who developed the K65R/S68G double mutation, restoration of the replication fitness of the virus through a compensatory mutation can help the virus survive under drug pressure, potentially leading to disease progression [33, 34]. Moreover, this adaptive evolution may shape HIV-1 at the population level, when these viruses contribute to epidemics through transmission. The resultant, potentially more virulent virus, could eventually modulate disease progression in newly infected hosts [35].

A limitation of this study is its small sample size. Owing to the high success rate of antiviral therapy in our cohort, only four patients with K65R/S68G double 
mutations were analyzed. Studies investigating a greater number of cases are warranted to elucidate the functional role of the S68G mutation. Nevertheless, this study provides evidence to aid the interpretation of DRMs in CRF01_AE strains.

\section{Conclusions}

We deciphered the role of the S68G mutation in the HIV RT of CRF01_AE strains. Our study provides new evidence for the interpretation of DRMs in the CRF01 AE strain and non-B subtypes of HIV-1. The present study also suggests the presence of additional potential DRMs in the non-B subtypes of HIV-1. Further studies are required to evaluate the involvement of the HIV phenotypes in the development of drug resistance.

\section{Supplementary information}

Supplementary information accompanies this paper at https://doi.org/10. 1186/s12879-020-4836-z.

Additional file 1: Figure S1. Flowchart of patients included in this study.

Additional file 2: Table S1. Prevalence of the S68G mutation among HIV-1 strains of various subtypes in the Stanford University HIV Drug Resistance Database.

Additional file 3: Table S2. Frequency of mutation on site 68 of reverse transcriptase between different subtypes in K65R mutants.

\section{Abbreviations}

3TC: Lamivudine; ART: Antiretroviral therapy; AZT: Zidovudine; DRMs: Drug resistance mutations; HIV: Human immunodeficiency virus; NRTI: Nucleotide/ nucleoside reverse transcriptase inhibitor; PCR: Polymerase chain reaction; TDF: Tenofovir

\section{Acknowledgements}

We would like to thank professor Hong Shang for her constructive suggestions for the design of this study and discussion on the manucript preparation. We also thank native English-speaking medical editors from the Charlesworth Group (http://charlesworth-group.com) for editorial assistance.

\section{Authors' contributions}

$\mathrm{SL}$ and $\mathrm{JO}$ designed and performed the experiments, analyzed data, and wrote the manuscript. BZ, MA, LW, HD, and MZ provided the data and information of the patients. $\mathrm{XH}$ conceived and designed the experiment, and revised the manuscript. All authors read and approved the final manuscript.

\section{Funding}

The National Natural Science Foundation (81871637) funded the design of the study and collection, analysis, and interpretation of data and in writing the manuscript.

\section{Availability of data and materials}

The datasets used and/or analyzed during the current study are available from the corresponding author upon reasonable request.

\section{Ethics approval and consent to participate}

The study protocol was approved by the Ethics Committee of the First Affiliated Hospital of China Medical University (Shenyang, China). Written informed consent was provided by all patients.

\section{Consent for publication}

Not applicable.

\section{Competing interests}

The authors declare that they have no competing interests.

\section{Author details}

${ }^{1} \mathrm{NHC}$ Key Laboratory of AIDS Immunology (China Medical University), Department of Laboratory Medicine, The First Affiliated Hospital of China Medical University, Shenyang 110001, China. ${ }^{2}$ National Clinical Research Center for Laboratory Medicine, Shenyang 110001, China. ${ }^{3}$ Key Laboratory of AIDS Immunology, Chinese Academy of Medical Sciences, Shenyang 110001, China. ${ }^{4}$ Department of Laboratory Medicine, The First Affiliated Hospital of China Medical University, Shenyang 110001, China.

Received: 18 September 2019 Accepted: 30 January 2020

Published online: 11 February 2020

\section{References}

1. Hemelaar J, Elangovan R, Yun J, Dickson-Tetteh L, Fleminger I, Kirtley S, Williams B, Gouws-Williams E, Ghys PD. Characterisation W-UNfHI: global and regional molecular epidemiology of HIV-1, 1990-2015: a systematic review, global survey, and trend analysis. Lancet Infect Dis. 2019;19(2): 143-55.

2. Kantor R, Katzenstein D. Drug resistance in non-subtype B HIV-1. J Clin Virol. 2004:29(3):152-9.

3. Snoeck J, Kantor R, Shafer RW, Van Laethem K, Deforche K, Carvalho AP, Wynhoven B, Soares MA, Cane P, Clarke J, et al. Discordances between interpretation algorithms for genotypic resistance to protease and reverse transcriptase inhibitors of human immunodeficiency virus are subtype dependent. Antimicrob Agents Chemother. 2006;50(2):694-701.

4. Brenner B, Turner D, Oliveira M, Moisi D, Detorio M, Carobene M, Marlink RG, Schapiro J, Roger M, Wainberg MA. A V106M mutation in HIV-1 clade C viruses exposed to efavirenz confers cross-resistance to non-nucleoside reverse transcriptase inhibitors. AIDS. 2003;17(1):F1-5.

5. Martinez-Cajas JL, Wainberg MA, Oliveira M, Asahchop EL, Doualla-Bell F, Lisovsky I, Moisi D, Mendelson E, Grossman Z, Brenner BG. The role of polymorphisms at position 89 in the HIV-1 protease gene in the development of drug resistance to HIV-1 protease inhibitors. J Antimicrob Chemother. 2012;67(4):988-94.

6. Holguin A, Sune C, Hamy F, Soriano V, Klimkait T. Natural polymorphisms in the protease gene modulate the replicative capacity of non-B HIV-1 variants in the absence of drug pressure. J Clin Virol. 2006:36(4):264-71.

7. Santos AF, Tebit DM, Lalonde MS, Abecasis AB, Ratcliff A, Camacho RJ, Diaz RS, Herchenroder O, Soares MA, Arts EJ. Effect of natural polymorphisms in the HIV-1 CRF02_AG protease on protease inhibitor hypersusceptibility. Antimicrob Agents Chemother. 2012;56(5):2719-25.

8. Gao F, Robertson DL, Morrison SG, Hui H, Craig S, Decker J, Fultz PN, Girard M, Shaw GM, Hahn BH, et al. The heterosexual human immunodeficiency virus type 1 epidemic in Thailand is caused by an intersubtype (A/E) recombinant of African origin. J Virol. 1996;70(10):7013-29.

9. Lau KA, Wong JJ. Current trends of HIV recombination worldwide. Infect Dis Rep. 2013;5(Suppl 1):e4.

10. Ode H, Matsuyama S, Hata M, Hoshino T, Kakizawa J, Sugiura W. Mechanism of drug resistance due to N88S in CRF01_AE HIV-1 protease, analyzed by molecular dynamics simulations. J Med Chem. 2007:50(8):1768-77.

11. Delviks-Frankenberry KA, Nikolenko GN, Maldarelli F, Hase S, Takebe Y, Pathak VK. Subtype-specific differences in the human immunodeficiency virus type 1 reverse transcriptase connection subdomain of CRF01_AE are associated with higher levels of resistance to 3'-azido-3'-deoxythymidine. J Virol. 2009:83(17):8502-13.

12. Tanuma J, Hachiya A, Ishigaki K, Gatanaga H, Lien TT, Hien ND, Kinh NV, Kaku M, Oka S. Impact of CRF01_AE-specific polymorphic mutations G335D and $A 371 \mathrm{~V}$ in the connection subdomain of human immunodeficiency virus type 1 (HIV-1) reverse transcriptase (RT) on susceptibility to nucleoside RT inhibitors. Microbes Infect. 2010;12(14-15):1170-7.

13. Taylor T, Lee ER, Nykoluk M, Enns E, Liang B, Capina R, Gauthier MK, Domselaar GV, Sandstrom P, Brooks J, et al. A MiSeq-HyDRA platform for enhanced HIV drug resistance genotyping and surveillance. Sci Rep. 2019; 9(1):8970.

14. Petropoulos CJ, Parkin NT, Limoli KL, Lie YS, Wrin T, Huang W, Tian H, Smith D, Winslow GA, Capon DJ, et al. A novel phenotypic drug susceptibility assay for human immunodeficiency virus type 1. Antimicrob Agents Chemother. 2000;44(4):920-8.

15. Hamilton MA, Russo RC, Thurston RV. Trimmed Spearman-Karber method for estimating median lethal concentrations in toxicity bioassays. Environ Sci Technol. 1977;11(7):714-9. 
16. Manocheewa S, Lanxon-Cookson EC, Liu Y, Swain JV, McClure J, Rao U, Maust B, Deng W, Sunshine JE, Kim M, et al. Pairwise growth competition assay for determining the replication fitness of human immunodeficiency viruses. J Vis Exp. 2015;99:e52610.

17. Monno L, Scudeller L, Brindicci G, Saracino A, Punzi G, Chirianni A, Lagioia A, Ladisa N, Lo Caputo S, Angarano G. Genotypic analysis of the protease and reverse transcriptase of non-B HIV type 1 clinical isolates from naive and treated subjects. Antivir Res. 2009;83(2):118-26.

18. Rhee SY, Varghese V, Holmes SP, Van Zyl GU, Steegen K, Boyd MA, Cooper DA, Nsanzimana S, Saravanan S, Charpentier C, et al. Mutational correlates of Virological failure in individuals receiving a WHO-recommended Tenofovircontaining first-line regimen: an international collaboration. EBioMedicine. 2017:18:225-35

19. Winters MA, Shafer RW, Jellinger RA, Mamtora G, Gingeras T, Merigan TC. Human immunodeficiency virus type 1 reverse transcriptase genotype and drug susceptibility changes in infected individuals receiving dideoxyinosine monotherapy for 1 to 2 years. Antimicrob Agents Chemother. 1997:41(4): 757-62.

20. Roge BT, Katzenstein TL, Obel N, Nielsen H, Kirk O, Pedersen C, Mathiesen L, Lundgren J, Gerstoft J. K65R with and without S68: a new resistance profile in vivo detected in most patients failing abacavir, didanosine and stavudine. Antivir Ther. 2003:8(2):173-82.

21. Doualla-Bell F, Avalos A, Brenner B, Gaolathe T, Mine M, Gaseitsiwe S, Oliveira M, Moisi D, Ndwapi N, Moffat H, et al. High prevalence of the K65R mutation in human immunodeficiency virus type 1 subtype $C$ isolates from infected patients in Botswana treated with didanosine-based regimens. Antimicrob Agents Chemother. 2006;50(12):4182-5.

22. McColl DJ, Chappey C, Parkin NT, Miller MD. Prevalence, genotypic associations and phenotypic characterization of K65R, L74V and other HIV-1 $\mathrm{RT}$ resistance mutations in a commercial database. Antivir Ther. 2008;13(2): 189-97.

23. Ross LL, Gerondelis $P$, Liao Q, Wine B, Lim M, Shaefer M, Rodriguez A, Gallant J, Lanier R. Selection for S68G/N/D mutations of HIV-1 reverse transcriptase (RT) in antiretroviral-Naïve subjects treated with Tenofovir (TDF)/Abacavir (ABC)/lamivudine (3TC) therapy. Abstr Interscience Conf Antimicrob Agents Chemother. 2005;45:272

24. Wirden M, Malet I, Derache A, Marcelin AG, Roquebert B, Simon A, Kirstetter M, Joubert LM, Katlama C, Calvez V. Clonal analyses of HIV quasispecies in patients harbouring plasma genotype with K65R mutation associated with thymidine analogue mutations or L74V substitution. AIDS. 2005;19(6):630-2.

25. Larder BA, Kohli A, Kellam P, Kemp SD, Kronick M, Henfrey RD. Quantitative detection of HIV-1 drug resistance mutations by automated DNA sequencing. Nature. 1993;365(6447):671-3.

26. Gibson RM, Schmotzer CL, Quinones-Mateu ME. Next-generation sequencing to help monitor patients infected with HIV: ready for clinical use? Curr Infect Dis Rep. 2014;16(4):401.

27. Svarovskaia ES, Feng JY, Margot NA, Myrick F, Goodman D, Ly JK, White KL, Kutty N, Wang R, Borroto-Esoda K, et al. The A62V and S68G mutations in HIV-1 reverse transcriptase partially restore the replication defect associated with the K65R mutation. J Acquir Immune Defic Syndr. 2008;48(4):428-36.

28. Garforth SJ, Lwatula C, Prasad VR. The lysine 65 residue in HIV-1 reverse transcriptase function and in nucleoside analog drug resistance. Viruses. 2014;6(10):4080-94.

29. Gu Z, Arts EJ, Parniak MA, Wainberg MA. Mutated K65R recombinant reverse transcriptase of human immunodeficiency virus type 1 shows diminished chain termination in the presence of 2',3'-dideoxycytidine 5'-triphosphate and other drugs. Proc Natl Acad Sci U S A. 1995;92(7):2760-4.

30. Shah FS, Curr KA, Hamburgh ME, Parniak M, Mitsuya H, Arnez JG, Prasad VR. Differential influence of nucleoside analog-resistance mutations K65R and L74V on the overall mutation rate and error specificity of human immunodeficiency virus type 1 reverse transcriptase. J Biol Chem. 2000; 275(35):27037-44

31. Garforth SJ, Domaoal RA, Lwatula C, Landau MJ, Meyer AJ, Anderson KS, Prasad VR. K65R and K65A substitutions in HIV-1 reverse transcriptase enhance polymerase fidelity by decreasing both dNTP misinsertion and mispaired primer extension efficiencies. J Mol Biol. 2010;401(1):33-44

32. Miller MD. K65R, TAMs and tenofovir. AIDS Rev. 2004;6(1):22-33.

33. Theys K, Deforche K, Vercauteren J, Libin P, van de Vijver DA, Albert J, Asjo B, Balotta C, Bruckova M, Camacho RJ, et al. Treatment-associated polymorphisms in protease are significantly associated with higher viral load and lower CD4 count in newly diagnosed drug-naive HIV-1 infected patients. Retrovirology. 2012;9:81.

34. Theys $K$, Abecasis AB, Vandamme AM. HIV-1 drug resistance: where do polymorphisms fit in? Future Microbiol. 2013;8(3):303-6.

35. Fraser C, Hollingsworth TD, Chapman R, de Wolf F, Hanage WP. Variation in HIV-1 set-point viral load: epidemiological analysis and an evolutionary hypothesis. Proc Natl Acad Sci U S A. 2007;104(44):17441-6.

\section{Publisher's Note}

Springer Nature remains neutral with regard to jurisdictional claims in published maps and institutional affiliations.
Ready to submit your research? Choose BMC and benefit from:

- fast, convenient online submission

- thorough peer review by experienced researchers in your field

- rapid publication on acceptance

- support for research data, including large and complex data types

- gold Open Access which fosters wider collaboration and increased citations

- maximum visibility for your research: over $100 \mathrm{M}$ website views per year

At BMC, research is always in progress.

Learn more biomedcentral.com/submissions 